\title{
Endoscopic Cricopharyngeal Myotomy for Management of Cricopharyngeal Achalasia in an 18-Month-Old Child
}

\author{
Robert Chun, MD; Mathew Sitton, MD; Neelesh A. Tipnis, MD; Joan C. Arvedson, PhD; \\ Aparna Rao, MD; Jason Dranove, MD; David J. Brown, MD
}

A 6-month-old patient presented with dysphagia and failure to thrive. Video fluoroscopic swallow study (VFSS), esophagogastroduodenoscopy, and manometry were diagnostic for CA. A gastrostomy tube was placed at 8 months. Botulinum toxin injection improved symptoms, but within 10 weeks symptoms returned.

At 18 months, an uncomplicated endoscopic CPM was performed. A postoperative VFSS demonstrated cricopharyngeal bar resolution. Within 3 months, patient was feeding orally without a G tube.

Pediatric CPA treatment options consist of dilation, botox, and transcervical CPM. To our knowledge, this is the youngest patient treated with endoscopic CPM. Intraoperative video and photographs are presented.

Key Words: Pediatric dysphagia, cricopharyngeal achalasia, endoscopic cricopharyngeal myotomy.

Laryngoscope, 123:797-800, 2013

\section{INTRODUCTION}

Cricopharyngeal achalasia $(\mathrm{CA})$ is a failure of the upper esophageal sphincter to open upon initiation of the pharyngeal swallow. CA is typically characterized by dysphagia, choking, and poor weight gain. Video fluoroscopic swallow studies (VFSS), manometry, clinical swallow evaluation, and endoscopic esophagoscopy are all useful in the diagnosis.

Treatment options vary from observation, botulinum toxin injection, mechanical dilation, and cervical cricopharyngeal myotomy $(\mathrm{CPM}) .^{1-4}$ In 2005, Baumen described the use of botox in an infant for CA. ${ }^{1}$ Messner recently described a series of seven botulinum toxin injections in three infants with $\mathrm{CA}^{2}{ }^{2}$ In this series, the

From the Department of Otolaryngology (M.s., R.c.), and Department of Gastroenterology (N.A.T.), and Division of Pediatric Gastroenterology (J.C.A.), Medical College of Wisconsin, [tk], Milwaukee, Wisconsin, U.S.A. Department of Child Health (A.R.), University of Arizona, Phoenix Children's Hospital, Phoenix, Arizona, U.S.A.; Department of Pediatric Gastroenterology (J.D.), Hepatology, and Nutrition, Levine Children's Hospital and Carolinas Medical Center, Charlotte, North Carolina, U.S.A.; Department of Otolaryngology-Head and Neck Surgery (D.J.B.), University of Michigan, Ann Arbor, Michigan, U.S.A. 4, 2012

Editor's Note: This Manuscript was accepted for publication June

Additional Supporting Information may be found in the online version of this article.

Poster Presentation: SENTAC, Kansas City, Missouri, Dec. 2011.

The authors have no funding, financial relationships, or conflicts of interest to disclose.

Send correspondence to Robert Chun, MD, 9000 West Wisconsin Ave., PO BOX 1997, Suite 550, Children's Hospital Clinics Building, Pediatric Otolaryngology Offices, Milwaukee, WI 53226.

E-mail: Rchun@mcw.edu

DOI: 10.1002/lary.23545 mean follow-up was 22 months. The time between botulinum toxin injections was on average every 3.3 months. The only complication was one hospital readmission for self-resolving aspiration.

Dilation of the upper esophageal sphincter has been described as a noninvasive treatment option for CA. Balloon dilation for CA was recently described for a 1-dayold newborn by Erdeve. ${ }^{1}$ Following four dilations, the patient's manometry and swallow were normal but follow-up was limited to 1 month after the last dilation. Other cases of balloon dilation have proceeded to surgical cricopharyngeal myotomy following limited benefit to dilation. ${ }^{3}$ While balloon dilation can improve CA, reoccurrence of symptoms may require surgical division of the cricopharyngeal muscle.

Cervical CPM was described by Brooks and colleagues in a series of five patients since $1976 .{ }^{4}$ In their series, patients presented from birth to 6 months with dysphagia and recurrent episodes of lower respiratory tract infections and failure to thrive. All patients did well without complications. They had good outcomes with long term follow-up (2-14 years). However, cervical cricopharyngeal myotomy does have associated risks including infection, hemorrhage, inadequate sectioning of the UES, transient unilateral vocal cord paralysis, recurrent laryngeal nerve damage, esophageal mucosa perforation, fistula formation, and mediastinitis. ${ }^{5}$

Endoscopic CPM was described by Pittman and Weissbrod, who discussed the surgical techniques and importance of leaving the buccopharyngeal fascia intact to prevent violation into the retropharyngeal space. ${ }^{6}$ Dauer and Brondo, with a separate series of patients, discussed the management of adult CA with endoscopic laser CPM, 


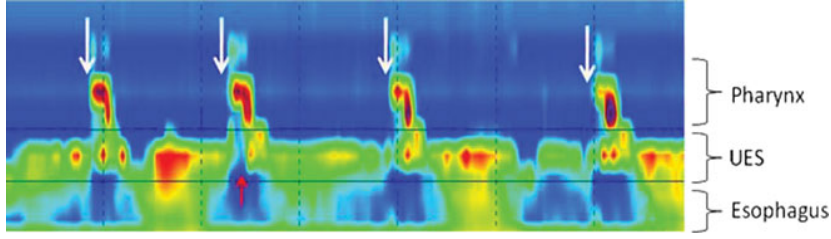

Fig. 1. Figure demonstrates high resolution esophageal manometry for our patient. The brackets demarcate the regions of the upper digestive tract: pharynx, upper esophageal sphincter (UES) and proximal esophagus. The colors represent contraction pressure: blue indicating low and red indicating high. The white arrows in the patients swallow indicate initiation of the swallow. The red arrow in the patients swallow indicates opening of the UES to allow passage of contents from the pharynx to the esophagus (blue channel). In this patient, the opening is brief and inconsistent (not seen in the other 3 swallows shown here).

symptomatic improvement, and no complications of mediastinitis. ${ }^{7-8}$

At the time of this publication, our case represents the youngest published patient to undergo endoscopic CPM in the treatment of CA. This case report will discuss multidisciplinary management in diagnosis and treatment.

\section{CASE REPORT}

A 6-month-old infant born at term presented to an outside institution with a lifelong history of choking with nipple feeds, and frequent respiratory illnesses requiring medical attention beginning in early infancy. This infant was initially bottle fed, but despite multiple formula changes, introduction of solids at 6 months of age, and aggressive gastroesophageal reflux treatment with proton pump inhibitors, his dysphagia continued and his weight fell below the third percentile by 8 months of age. The patient otherwise met all developmental milestones for his age. There were no signs of a global neuromuscular or central nervous system abnormality. The patient had a normal MRI of brain, ruling out a Chiari malformation, which can be a cause for pediatric dysphagia. Videofluoroscopic swallow studies (VFSS) had been completed at outside institutions, according to the parents. They stated that their child aspirated on liquid with bottle feedings as an infant and on puree with spoon feeding beginning about 6 months of age. An esophagogastroduodenoscopy revealed difficulty intubating the upper esophageal sphincter, necessitating change to a neonatal endoscope, which did pass with success; biopsies performed revealed normal esophageal mucosa. This supported the suspicion of CA. Due to continued dysphagia, intermittent aspiration, and inadequate weight gain, a gastrostomy tube was placed at 8 months. At 11 months of age, this infant underwent botulinum toxin injection into the cricopharyngeal musculature. A repeat VFSS showed resolution of the cricopharyngeal bar and no aspiration, with significant clinical improvement. Unfortunately within 10 weeks his dysphagia returned, repeat VFSS showed return of CA, and he resumed his NPO status except for minimal tastes of puree by spoon.
The patient's care was transferred to the Children's Hospital of Wisconsin at 16 months of age. He was evaluated in the interdisciplinary aerodigestive and voice clinic with an otolaryngologist, gastroenterologist, pulmonologist, and speech-language pathologist. These professionals consult together to diagnose, manage, and treat patients in an interdisciplinary fashion with one visit for the patient and family. The patient underwent a coordinated EGD, bronchoscopy, and endoscopic CPM following the initial clinic visit.

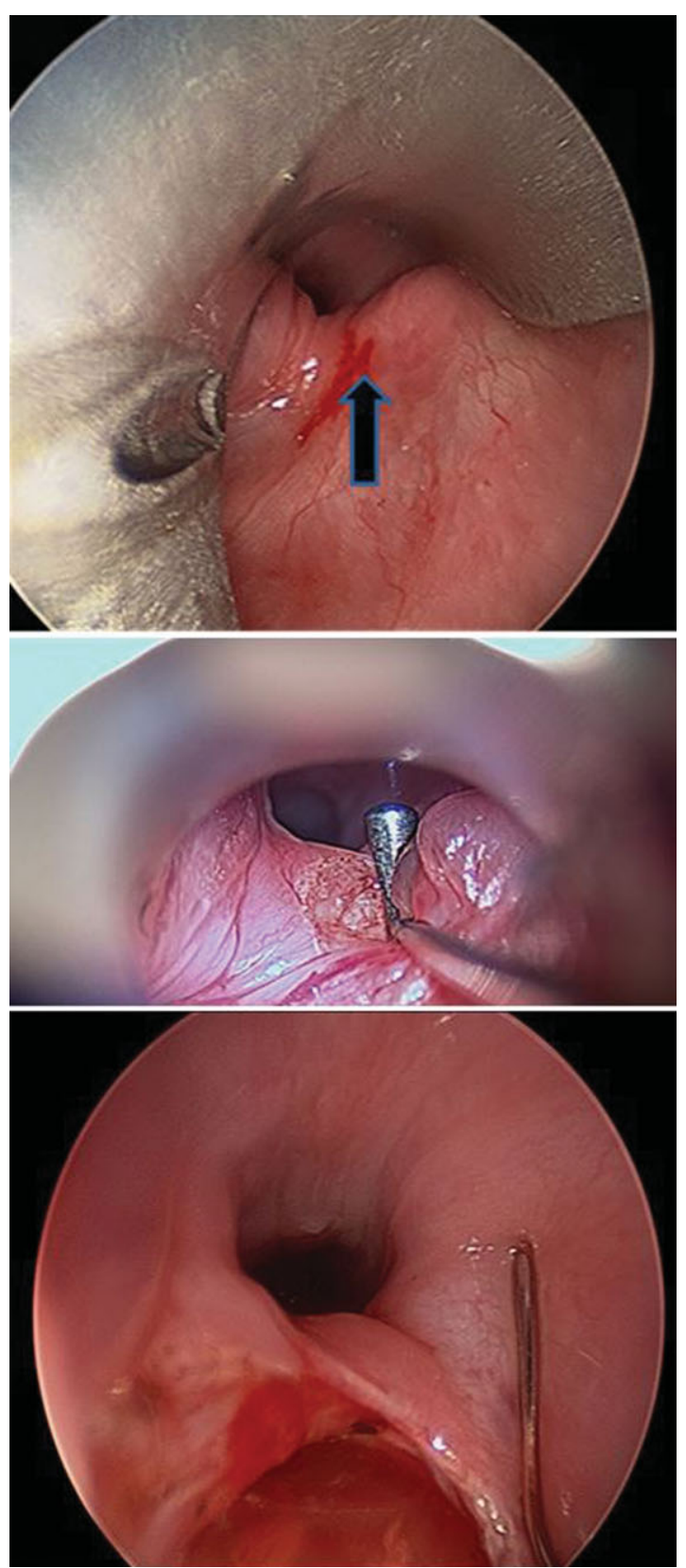

Fig. 2. Top Panel: Microscopic view of cricopharyngeal bar under suspension. Arrow demonstrates cricopharyngeal muscle; Middle Panel: Laser resection of muscle fibers of cricopharyngeus muscle with counter retraction with laryngeal probe; Lower Panel: Completed cricopharyngeal myotomy with resection to buccopharyngeal fascia with visualization of fat and nodes in retropharyngeal space. 
Fig. 3. Left Panel: Preoperative VFSS demonstrating cricopharyngeal bar; Right Panel: Resolution of cricopharyngeal bar.
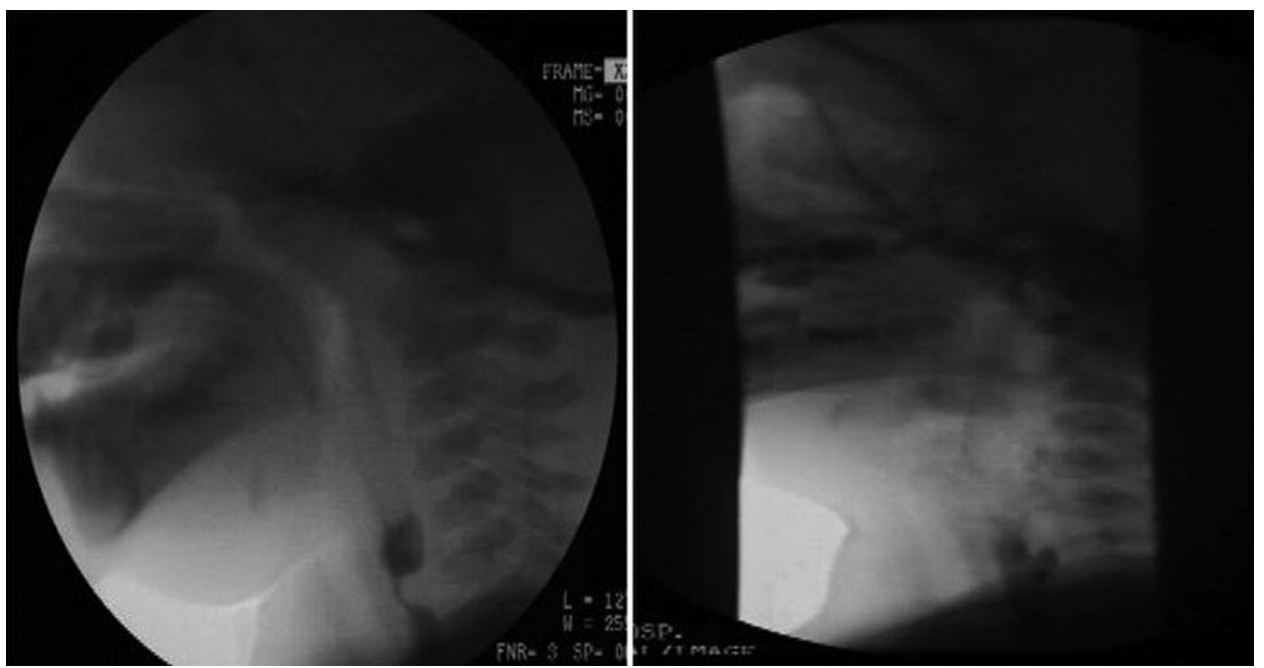

A video fluoroscopic swallow study (VFSS) was performed with findings consistent for CA. The child was seated on the Multiple Application Multiple Articulation (MAMA) chair in upright position for a lateral view of oral, pharyngeal, and upper esophageal phases of swallowing. The child's mother presented a spoon with very thin liquid (apple juice, 4 presentations), thickened liquid (nectar consistency apple juice, 3 presentations), and pureed food (pudding, 3 presentations). Child was calm and cooperative, until the final presentation of pudding when he fussed. Findings were reviewed with parents by a speech-language pathologist (SLP) and radiologist. The findings were then reviewed independently by two SLPs (7 and 13 years experience in conducting, reading, and interpreting VFSS with pediatric patients) with consensus reached for objective findings.

In summary, the child demonstrated functional bolus formation and oral transit. A borderline delay in pharyngeal swallow initiation was noted only rarely when material was seen in the valleculae and pyriform sinuses prior to initiation of a pharyngeal swallow. Trace silent aspiration was seen just to the underside of the vocal folds on very thin liquid, which was ejected from the airway upon exhalation. Thickened liquid and puree were seen along the lower posterior pharyngeal wall, in pyriform sinuses, and on the upper esophageal sphincter (UES) following swallows when the boluses did not move completely through the UES. Aspiration occurred on the residue occasionally with no cough. The primary finding in the upper esophagus was a prominent cricopharyngeal bar that did interfere with bolus passage at times. The child produced additional "dry" swallows and cleared the pharynx of the residue which moved through the esophagus. He had no deficits in tongue base retraction with no residue in the valleculae post swallows. The pharyngeal residue related more directly to upper esophageal phase than to pharyngeal phase swallowing with a small amount of residue in the lower pharynx. Retrograde movement was noted occasionally from the esophagus up into the pharynx.

The endoscopic CPM was performed with microsuspension laryngoscopy with exposure of the cricopharygeal muscle by suspending a parson's laryngoscope posterior to the larynx. An orogastric tube was placed to facilitate laryngoscopic positioning and exposure and was removed prior to lasering. $\mathrm{CO}_{2}$ laser was used at a setting of 7 watts, scan mode with a $0.7-\mathrm{mm}$ size spot with a $0.3 \mathrm{sec}-$ ond delay. An angled laryngeal retractor was used to provide counter tension on the muscle fibers to facilitate dissection. Layer by layer dissection was performed until the gray translucent buccopharyngeal fascia was visualized with the underlying fat and nodes of the retropharyngeal space could be seen.

A postoperative VFSS within 24 hours after the procedure demonstrated resolution of the cricopharyngeal bar, and only one trace occasional minimal aspiration just prior to the second swallow of very thin liquid, due to slight delay in initiating a pharyngeal swallow. A small amount of residue was seen in the pharynx, which was much less than preoperatively, and the child cleared the residue with a follow-up swallow. A pharyngeal defect was noted secondary to the CPM, and the patient developed no signs or symptoms of infection. Nine months post surgery, the patient has had no recurrence of symptoms, his gastrostomy tube has been removed, he is taking a regular diet with no restrictions, and his weight is steady at the 50th percentile.

\section{DISCUSSION}

Cricopharyngeal achalasia (CA) is an uncommon cause of dysphagia in neonatal and pediatric populations. The etiology of CA is likely idiopathic and is secondary to a failure of the upper esophageal sphincter (UES) to relax and open sufficiently for a bolus to pass through the UES during a swallow. ${ }^{1}$

Upper esophageal endoscopy identified a narrow cricopharyngeal luminal diameter. While positive response to previous botulinum toxin suggests CA, prior to a definitive operative treatment, manometric studies can functionally confirm a diagnosis of CA. High-resolution esophageal manometry was performed and noted both elevated resting pressure $(70 \mathrm{mmHg})$ and poor relaxation of the cricopharyngeal musculature (See Fig. 2). 
Pharyngeal contraction pressure and esophageal body function were normal, which is an important finding to support a positive prognosis for oral feeding gains anticipated following surgery. Manometry demonstrated a bolus permissive period of only about 0.1 second, with normal about $0.3-0.4$ seconds (in adults) in duration. ${ }^{9}$ These findings are consistent with CA. Previous MRI of the brain failed to identify posterior fossa abnormalities that can be associated with CA.

Upper esophageal endoscopy ruled out concomitant diseases such as reflux esophagitis and eosinophilic esophagitis (EoE) via biopsy of the esophagus. GER has been shown to cause hyperactive cricopharyngeus muscle and is important to aggressively treat prior to CPM. ${ }^{10-12}$ Furthermore, untreated EoE may complicate postoperative swallowing and healing as it is known to complicate aerodigestive procedures such as laryngotracheal reconstruction outcomes. ${ }^{13}$

Due to this patient's history of aspiration and RSV bronchiolitis, there were long-term concerns for his respiratory morbidity with continued feeding difficulty and aspiration. A flexible bronchoscopy was performed and revealed no anatomical abnormalities.

Botulinum toxin only temporarily resolved the child's symptoms. Parents expressed a desire for definitive long-lasting treatment, surgical options were considered. These options included external versus endoscopic CPM. In contrast to the risks of transcervical cricopharyngeal myotomy, the risks of endoscopic cricopharyngeal myotomy approach include restenosis, retropharyngeal space infection and mediastinitis, thermal injury, fistula formation, and subcutaneous emphysema. Endoscopic CPM offers a minimally invasive approach without risk of laryngeal nerve injury, but possibly has higher risk of retropharyngeal space violation and infection. Endoscopic CPM may possibly provide definitive treatment under a single anesthetic, while other methods of dilation and botulinum toxin may take repetitive procedures for treatment.

The endoscopic CPM was performed with $\mathrm{CO}_{2}$ laser. Intraoperative and postoperative antibiotics and proton pump inhibitors were given following the procedure. Decadron $(0.5 \mathrm{mg} / \mathrm{kg})$ was given once due to concerns for post op laryngeal edema. The risks and benefits of intraoperative steroid use should be considered in each individual case, weighing benefits of decreasing laryngeal edema versus disadvantages of immunosuppression and hindrance to wound healing. It is essential to keep the buccopharyngeal fascia layer intact to prevent violation into the retropharyngeal space and possible subsequent infection. The buccopharyngeal is a glistening gray fascia that is discernable from the overlying cricopharyngeal muscle and from the underlying fat of the retropharyngeal space. Because the retropharyngeal space descends into the anterior mediastinum, violation of the buccopharyngeal fascia can possibly lead to mediastinal infection.

The child's follow-up VFSS was completed about 24 hours after the myotomy (Fig. 7). A cricopharyngeal bar was not prominent. Boluses moved through the UES with no restriction. Trace aspiration on very thin liquid was noted one time just prior to initiation of a pharyngeal swallow, due to a timing problem and not a cricopharyngeal deficit. A small "pouch" was noted at the posterior UES, with a small amount of barium contrast going retrograde in the submucosal space between the mucosa and buccopharyngeal fascia, but no barium entered or descended into the retropharygeal space distally. This may be due to the barium reaching the lower limit of the dissection and "refluxing" up into the submucosal space superiorly. The fact that the retropharyngeal space is a true and not potential space in children may also explain why the barium refluxed superficial to the buccopharyngeal fascia and retropharyngeal space. Follow-up VFSS 2, 4, and 6 months after procedure showed no signs of restenosis.

\section{CONCLUSION}

Infants and children with cricopharyngeal achalasia have multiple factors underlying their dysphagia, not simply upper esophageal sphincter dysfunction. While botox, dilation, and cervical CPM may be used for treatment of CA symptoms, our case demonstrates that endoscopic CPM is possible in the age group and can successfully resolve symptoms. While a large surgical cohort would be ideal to access morbidity, long-term success, and complications of pediatric endoscopic CPM, our case report suggests that endoscopic CPM may be performed with low morbidity and decreased anesthesia exposure compared to other methods. Long-term safety and efficacy in treatment is unknown until larger samples of pediatric endoscopic CPM can be performed and followed.

\section{BIBLIOGRAPHY}

1. Sewell RK, Baumen N. Congenital cricopharyngeal achalsia: management with botulinum toxin before myotomy. Arch Oto Head and Neck Surgery 2005; $131: 451-453$

2. Barnes MA, Ho AS, Malhotra PS, Koltai P, Messner A. The use of botulinum toxin for pediatric cricopharyngeal achalsia. IJPO 2011;75: $1210-1214$.

3. Mathur NB, Banerjee S, Maria A, Bhatnagar V. Congenital cricopharyngeal achalsia. Indian Pediatr 2001;38:783-788

4. Brooks Millar A, Rode H. The surgical management of cricopharyngeal achalasia in children. IJPO 2000;56(1):1-7.

5. De Caluwe D, Nassogne MC, Reding R, de Ville de Goyet J, Clapuyt P, Otte JB. Cricopharyngeal achalsia: case report and review of the literature eur. J Pediatric 1999;9:109-112.

6. Pittman M, Weissbrod P. Endoscopic CO2 laser cricopharygneal myotomy. Laryngoscope 2009;119:45-53.

7. Dauer E, Salassa J, Iuga L, Kasperbauer J. Endoscopic laser vs open approach for cricopharyngeal myotomy. Otolaryngol Head Neck Surg 2006;134:830-835

8. Brondbo K. Treatment of cricopharyngeal dysfunction by endoscopic laser myotomy. Acta Otolaryngol 2000;Suppl 543:222-224.

9. Gosh SK, Pandolfino JE, Zhang Q, Jarosz A, Kahrilas PJ. Degluttative upper esophageal sphincter relaxtion: a study of 75 volunteer subjects using solid state high resolution manometry. Am J Physiol Gastrointest Liver Physiol 2006;291:G525-531

10. Ekberg O, Lindgren S. Gastroesophageal reflux and pharyngeal function. Acta Radiol Diagn 1986;27:421-423.

11. Hunt PS , Connell AM,Smiley,TB. The cricopharyngeal sphincter in gastric reflux, Gut 1970;11:303-306.

12. Sondheimer JM. Upper esophageal sphincter and pharyngoesophageal motor function in infants with and without gastroesophageal reflux. Gastroenterology 1983;85:301-305.

13. Hartnick CJ, Liu, JH, Cotton RT, Rudolph C. Subglottic stenosis complicated by allergic esophagitis: case report. Ann Otol Rhinol Laryngology 2002;111(1):57-60 